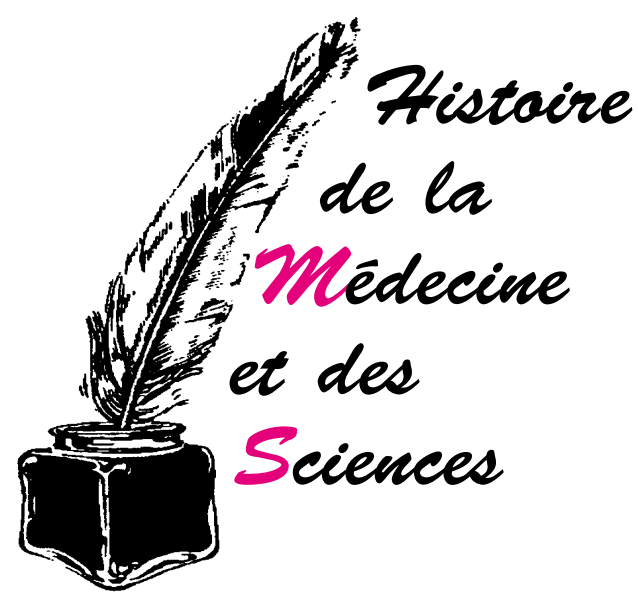

\title{
Sexualité et procréation: problème de frontière
}

\section{Réflexion autour de la "querelle de l'interstitiel" des années 1920, ou la découverte de la glande de la masculinité (1903-1930)}

V ouloir tracer une frontière entre sexualité et procréation ne va pas sans soulever des critiques d'ordre scientifique, socio-politique, idéologique, théologique et éthique. Aujourd'hui, d'un côté de la frontière, le Sida, pandémie à haut risque pour l'homme, implique une sexualité sans procréation et, de l'autre côté, le développement des techniques et des pratiques des procréations médicalement assistées (PMA) conduisent l'homme à ce pouvoir biomédical de procréer sans sexualité. Si les préservatifs sont nécessaires et les PMA des actes médicaux utiles, des groupes sociaux et des personnes, défendant des structures idéologiques ou une philosophie théologique, s'appuyant sur la science ou la «morale», s'élèvent contre de telles nécessités ou de telles utilités. Aussi les médias ne manquent-ils pas d'informer le public des débats touchant aussi bien les pharmaciens qui refusent pour des raisons de morale religieuse de vendre des préservatifs, arguant exclusivement une finalité procréatrice de la sexualité ; des scandales soulevés autour des "mères grand-mères ${ }^{(1)}$ : avoir un enfant pour une femme de 65 ans peut poser des problèmes sociaux, éthiques et surtout humains; ou encore des scénarios catastrophes qui brandissent les dérives eugéniques que pourront
Notre histoire contemporaine n'a jamais été aussi ouverte à de tels débats touchant la reproduction et la sexualité humaines. Toutefois, la dispute n'est pas nouvelle. Dans les années 20, une querelle, connue sous la dénomination de «querelle de l'interstitiel»(2) [1] s'est déroulée au sein des milieux biologiques et médicaux français, à propos du rôle joué par les hormones dans la détermination du sexe.

Cette querelle a essentiellement son origine dans les travaux de Pol Bouin et Paul Ancel de 1903 à la fin des années 20. Tous deux étaient médecins mais délaissèrent la pratique médicale pour se consacrer à l'histophysiologie et à l'embryologie. Dans sa thèse de médecine Bouin (1897) observa que, chez le cobaye, les testicules expérimentalement atrophiés présentent une absence des éléments de la lignée séminale et une augmentation des cellules interstitielles, cellules formant un tissu diffus entre les tubes séminifères. Si la thèse de

(1) C'est la traduction de l'expression employée par la presse italienne de la "mamma nonna», voir p.e. La Republica du mardi 4 avril 1995 l'article «E la mamma-nonna sfida "Io voglio un altro figlio" " ( Et la mère grand-mère défie "Je veux un autre enfant" ").

${ }_{(2)}^{(2)}$ Le tissu interstitiel est formé de cellules sécrétrices groupées en îlots entre les tubes séminifères et parfois disposées autour des vaisseaux sanguins testiculaires. Ces cellules ont été découvertes par Leydig en 1850 et constituent la glande endocrine du testicule produisant la «testostérone». médecine soutenue par Ancel (1899) concernait un sujet anatomique (étude du péritoine), sa thèse de doctorat ès sciences naturelles (1903) fut consacrée à l'histogenèse sexuelle chez l'escargot. Bouin et Ancel avaient des intérêts scientifiques communs et leur collaboration répondait à une logique intellectuelle.

Le père de Bouin était vétérinaire. Il signala à son fils le comportement "singulier des chevaux pifs", ou cryptorchides, c'est-à-dire des chevaux dont les testicules sont restés en position intra-abdominale (testicules ectopiques). Ces chevaux montrent une vigueur sexuelle exceptionnelle et deviennent "méchants", nécessitant l'intervention du vétérinaire pour les castrer. Paradoxalement, ces chevaux "pifs», comme les verrats «riles", sont stériles. Bien connue des vétérinaires, cette dissociation entre un comportement génésique normal et une stérilité due à l'absence des lignées séminales ne cessait, selon l'expression de Robert Courrier, de «hanter» l'esprit de Pol Bouin.

Ensemble à la faculté de médecine de Nancy, puis à partir de 1918-1919 à celle de Strasbourg, Bouin et Ancel contribuèrent dans une collaboration intime et longue à la connaissance et au développement de la biologie sexuelle. Ils fondèrent une école et formèrent de nombreux élèves dont trois se distingueront dans cette 
discipline et obtiendront une chaire au Collège de France: Robert Courrier (endocrinologie, 1938), Jacques Benoît (histophysiologie, 1952) et Étienne Wolff (embryologie, 1955).

Les premières publications de Bouin et Ancel sur le tissu interstitiel datent de 1903, dont une synthèse "Recherche sur les cellules interstitielles du testicule des mammifères ". Dans cette synthèse, Bouin et Ancel présentaient une étude détaillée de ces cellules au cours de l'embryogenèse, puis chez le jeune, l'adulte et aussi dans le cas particulier de la cryptorchidie. Ils confirmèrent l'origine conjonctive de ces cellules, puis établirent qu'il existe chez l'embryon une indépendance entre le tissu interstitiel et les structures séminales: il s'agit d'un point capital de leur recherche qu'ils résumaient par ces phrases : «L'appareil interstitiel atteint tout son perfectionnement morphologique à un stade de l'ontogenèse où la glande séminale présente encore tous ses caractères embryonnaires. Elle se développera encore, mais elle ne changera plus d'aspect. Il existe donc une indépendance évidente entre le développement de l'appareil interstitiel et celui de la glande séminale. » (1903, p. 463.) En 1901, Claudius Regaud et Albert Policard de l'école histophysiologique de Lyon avaient déjà observé cette indépendance sans pousser leur recherche au-delà de la simple observation.

Bouin et Ancel, poursuivant leurs investigations morphohistologiques et expérimentales, démontrèrent que les cellules interstitielles correspondent à la glande mâle et qu'elles peuvent être responsables de la morphogenèse des caractères sexuels secondaires, voire primaires (1904 et 1906). Après avoir montré le rôle joué par le corps jaune gestatif dans la mise en place de la «dentelle utérine » préparant la nidation de l'œuf (1910) et essayé, en vain, d'extraire le produit actif du tissu interstitiel, Bouin et Ancel ne reprirent leurs travaux qu'après la fin de la guerre et la réorganisation de l'université de Strasbourg.

Dans ce problème particulier de la détermination du sexe, les travaux de Mac Clung (1902) et ceux de Carl
Correns (1907) sur les chromosomes dits «sexuels» (hétérochromosomes) et leur nomination par les lettres $\mathrm{X}$ et Y par Edmund-Beecher Wilson en 1911 ne devaient pas pour autant conduire tous les biologistes vers une idée d'une détermination génétique stricte de la différenciation sexuelle. Si Thomas-Hunt Morgan et son école considéraient (avec des nuances) que les chromosomes $\mathrm{X}$ et $\mathrm{Y}$ jouent un rôle qualitatif dans la détermination du sexe, d'autres pensaient que ces chromosomes, modifiant le rapport nucléo/plasmatique de la cellule, ont une action trophique déterminant le sexe. Quant à une détermination «hormonale» du sexe, elle gardait de nombreux partisans, d'autant plus que les travaux de l'école embryologique de Chicago, dirigée par Franck-Rattray Lillie, avaient, depuis 1918, redonné une nouvelle vigueur à ces recherches avec la découverte du Free-martinisme ${ }^{(3)}$.

Les opposants à l'école de BouinAncel vont se manifester avec vigueur dès le début des années 20. A leur tête Serge Voronoff, chirurgien reconnu internationalement (en particulier dans le domaine des greffes osseuses) et ses collaborateurs P. Dartigues (médecin chirurgien) et Édouard Retterer (histologiste). Ils sont appuyés par Eugène Gley, professeur de physiologie au Collège de France accompagné de son élève Albert Pézard et par Christian Champy (Faculté de médecine de Paris). Ces médecins biologistes rejettent l'idée d'une quelconque activité du tissu interstitiel pouvant jouer un rôle dans la physiologie sexuelle. Ils avancent même que le tissu interstitiel est absent du testicule des mammifères. En revanche, ils accordent aux cellules de Sertoli qui entourent les tubes séminifères et qui paraissent avoir une action sur la spermatogenèse (rôle trophique), une activité sécrétrice «humorale» (harmozone de Gley) responsable de l'activité sexuelle et de la fonction reproductrice $^{(4)}$.

L'équipe de Voronoff avait tout intérêt à défendre cette idée car, héritière des Brown-Séquard et Steinach ${ }^{(5)}$ $[4,5]$, elle lançait tout un programme de greffes testiculaires de singe (babouins) chez l'homme ayant per- du ses forces en raison de l'âge. Redonner de la vigueur aux hommes âgés pour leur permettre non seulement de reprendre une activité intellectuelle intense mais aussi de procréer de nouveau faisait partie du plan idéologique de cette équipe ouverte au développement d'un eugénisme positif. Aussi, quand un greffon de babouin greffé dans la vaginale testiculaire d'un homme pourrait être récupéré, il était soumis au diagnostic histologique de Retterer. Celui-ci ne voyait aucune cellule interstitielle mais reconnaissait (dans ces structures nécrosées) des cellules de Sertoli autour de tubes séminifères vides de spermatozoïdes. Or, comme les hommes greffés (environ 500 entre 1920 et 1930) reprenaient plus ou moins de forces selon les cas, la démonstration du rôle des cellules de Sertoli paraissait être faite aux dépens du tissu interstitiel.

C'est en 1924, lors de la $19^{\text {e }}$ réunion de l'Association des anatomistes qui se tenait à Strasbourg que la «querelle de l'interstitiel» vécut un de ses moments les plus violents: Bouin et Ancel défendaient leur conception contre Champy, porte-parole de l'équipe parisienne. Cette querelle se déroulait dans un contexte historique qu'il faut préciser pour en comprendre le fond. 1918, c'est l'année de la fin de la Première

\footnotetext{
(3) Les "Free-Martin" sont des jumeaux bovins mâle et femelle, cette dernière ayant été masculinisée par son jumeau mâle à la suite d'une fusion placentaire et d'une anastomose des vaisseaux sanguins. Les "Free-Martin» représentaient une expérience naturelle qui parlait en faveur d'une théorie "humorale» d'une différenciation sexuelle ou suivant l'expression souvent employée par les biologistes d'un "déterminisme du sexe».

(4) Le syncytium de Sertoli joue un rôle trophique pendant la différenciation des spermatogonies. Les cellules de Sertoli auraient une fonction sécrétrice mais seules les cellules interstitielles, ou cellules de Leydig, sécrètent l'hormone sexuelle mâle (testostérone) [2, 3]. (5) Brown-Séquard s'était à la fin des années 1880 injecté du "suc" de testicules de cobaye et avait noté une amélioration de ses forces physiques et intellectuelles: c'était la cure de jouvence par l'opothérapie $($ opos $=s u c)$. Quant à E. Steinach, il pensait pouvoir «rajeunir» des organismes vieillissants en employant la méthode de résection des canaux déférents (méthode de Bouin et Ancel), qui permettait un développement de la glande interstitielle et une augmentation de la sécrétion d'hormone mâle. Il réalisa l'expérience sur des rats séniles qui, après l'opération reprenaient une "activité normale». L'idée sera reprise pour être appliquée à l'homme.
} 
Guerre mondiale qui a fait, rien que pour la France, 1482000 morts. 1917-1920, c'est aussi l'époque de l'épidémie de grippe espagnole qui sera de par le monde responsable de plusieurs millions de morts, c'est aussi la famine... Aussi, une politique de natalité devenait-elle prioritaire. Alors, comment accepter l'idée d'un système biologique dissociant la sexualité de la procréation: il y avait là une contradiction éthique qui s'opposait à une politique de repeuplement en général et qui contrariait les projets idéologiques (et rentables) de l'équipe de Voronoff en particulier $^{(6)}$ [7].

Partant d'une étude sur un problème de physiologie sexuelle (testicules ectopiques), Bouin et Ancel posaient l'hypothèse du rôle fondamental que devaient jouer les "hormones sexuelles » dans la différenciation, voire la détermination, du sexe. A la suite des travaux des chimistes et biochimistes des années 1920, les premières synthèses d'hormones sexuelles furent faites au début des années 1930 ; les embryologistes démontrèrent alors le rôle morphogène de ces hormones sur la différenciation sexuelle. L'hypothèse de Bouin et Ancel posée en 1903 recevait une démonstration expérimentale en 1935. Aussi, tous les travaux sur l'hormonologie sexuelle prennent-ils une source historique dans les premières investigations élaborées par Bouin et Ancel au début du siècle ${ }^{(7)}$.
L'idée d'une substance humorale responsable du développement et du fonctionnement des organes génitaux, indépendante d'une maturation et d'une activité des cellules reproductrices, souleva de vives critiques dont le fond socio-politique et idéologique est évident après 1918. Aujourd'hui, le débat sur la «sexualité » et les PMA est plus complexe dans le fond que celui concernant la "glande de la masculinité " des années 1920, mais la forme est la même dans la mesure où la question est celle posée par une séparation entre sexualité et procréation

(6) A. Lipschutz en 1946 évoque ce souvenir lors $d u$ jubilé de P. Bouin: "Je me rappelle les années qui se passèrent entre 1920 et 1930 environ. La théorie interstitielle fut discutée dans des centaines de travaux et elle fut très combattue. On se faisait célèbre en la combattant! Aujourd'hui - quinze ans plus tard seulement - personne ne voudra se rappeler ces écrits. " Lipschutz souligne dans son texte que dans ces années 20, pendant lesquelles se développait une politique de repeuplement, une théorie qui dissociait une glande endocrine responsable des fonctions génitales de la "glande générative", c'est-à-dire de la fonction de reproduction, avait quelque chose de "gênant»: pour lui, c'était là un aspect «socio-psychologique» qui doit nous rendre cette attitude compréhensible du point de vue "subjectif».

(7) Toutefois, Bouin et Ancel avançaient que chez l'embryon «l'hormone» mâle orientait les gonades et les cellules sexuelles indifférenciées vers le sexe mâle. C'était là leur volonté de faire dépendre une détermination primaire du sexe par cette "substance» sécrétée par les cellules interstitielles.

\section{RÉFÉRENCES}

1. Fischer JL. Les recherches sur l'intersexualité expérimentale chez les vertébrés dans l'école française de l'entre-deuxguerres. In: Debru C, Gayon J, Picard JF, eds, Les Sciences biologiques et médicales en France 1920-1930. Paris: Cnrs éditions: 1994: 163-75

2. Jégou B. La cellule de Sertoli : actualisation du concept de cellule nourricière. $m e ́-$ decine/sciences 1995; 11: 519-27.

3. Saez J, Lejeune H, Avallet O, Habert R, Durand P. Le contrôle des fonctions différenciées de la cellule de Leydig. médecine/sciences 1995; 11: 547-54.

4. Brown-Séquard CE. Nouveaux faits relatifs à l'injection sous-cutanée, chez l'homme, d'un extrait de testicules mammifères. Arch Physiol Norm Pathol 1890; 22: 201-8.

5. Fischer JL. Histoire du déterminisme épigénétique du sexe chez les animaux et l'homme de 1800 à 1935. Thèse de doctorat d'État ès lettres, Université de Paris-Panthéon-Sorbonne, 1993, $740 \mathrm{p}$.

6. Lipschutz A. Discours, in Jubilé de Monsieur le Professeur Pol Bouin (18 novembre 1946). Arch Histol Embryol 1947; 30 : 109-40.

\section{Jean-Louis Fischer}

Centre Alexandre-Koyré, Histoire des Sciences et des Techniques, EHESS-Cnrs (UMR 48), Muséum National d'Histoire Naturelle, Pavillon Chevreul, 57, rue Cuvier, 75231 Paris Cedex 05, France. 